\title{
Stop codons and the +4 nucleotide may influence the efficiency of G418 in rescuing nonsense mutations of the HERG gene
}

\author{
HAIYUN YU ${ }^{1 *}$, YANHAI MENG ${ }^{2 *}$, SHUHONG ZHANG $^{3}$, CHEN TIAN $^{4}$, FANG WU $^{4}$, \\ NING LI ${ }^{4}$, QIUYANG $\mathrm{LI}^{4}$, YULAN JIN ${ }^{1}$ and JIELIN PU ${ }^{5}$ \\ ${ }^{1}$ Department of Pathology, Beijing Obstetrics and Gynecology Hospital, Capital Medical University, \\ Beijing 100006; ${ }^{2}$ Department of ICU, Fuwai Hospital, National Center for Cardiovascular Diseases, \\ Chinese Academy of Medical Sciences and Peking Union Medical College, Beijing 100037; ${ }^{3}$ Department of Pathology, \\ Beijing Friendship Hospital, Capital Medical University, Beijing 100050; ${ }^{4}$ Department of Pathology, \\ Capital Medical University Electric Power Teaching Hospital (State Grid Corporation Beijing Electric Power Hospital), \\ Beijing 100037; ${ }^{5}$ Department of Cardiology, East Hospital, Tongji University, Shanghai 200120, P.R. China
}

Received March 24, 2019; Accepted August 16, 2019

DOI: 10.3892/ijmm.2019.4360

\begin{abstract}
The importance of the local sequence context in determining how efficiently aminoglycosides rescue nonsense mutations has been established previously in disease models. Different stop codons appear to facilitate the termination process with differing efficiencies. Furthermore, the efficiency with which termination is suppressed may also be influenced by the local sequence context surrounding the stop codon. The strongest bias has usually been identified with the nucleotide base that immediately follows the stop codon in the majority of experiments. However, how the sequence context influences the efficiency of aminoglycosides in rescuing the human ether-a-go-go-related (HERG) protein in mammalian cells remains to be fully elucidated. Therefore, the present study was devised to examine the susceptibility of different termination codons on the HERG gene and the +4 nucleotide immediately following them to be suppressed by aminoglycosides in 293 cells. The 293 cells were transiently transfected with the wild-type or mutant genes. The read-through effect was subsequently examined by adding aminoglycoside G418 into the culture medium, followed by incubation of the cells
\end{abstract}

Correspondence to: Professor Jielin $\mathrm{Pu}$, Department of Cardiology, East Hospital, Tongji University, 150 Gimo Road, Pudong, Shanghai 200120, P.R. China

E-mail: jielinpu1@163.com

Professor Yulan Jin, Department of Pathology, Beijing Obstetrics and Gynecology Hospital, Capital Medical University, 17 Qihelou Street, Dongcheng, Beijing 100006, P.R. China

E-mail: jinyulan.love@163.com

*Contributed equally

Key words: aminoglycoside, nonsense mutation, stop codon, inherited arrhythmia, sudden cardiac death for $24 \mathrm{~h}$. An immunofluorescence method was then used to observe the protein expression of HERG prior to and following drug treatment. Patch clamping was performed to evaluate the function of the HERG protein. These experiments revealed that stop codons TGA and TAA in the R1014X mutant were more susceptible to treatment with the drug G418. Similar results were observed with the W927X-TGA and W927X-TAA mutants. Subsequently, R1014X-TGAC, R1014X-TGAG and R1014X-TGAA mutants were constructed based on the R1014X-TGAT mutant. The level of red fluorescence was observed prior to and following the administration of G418 using antibodies targeting the $\mathrm{N}$ - or $\mathrm{C}$-terminus of the HERG protein. However, the tail current density was found only to increase with the R1014X-TGAT mutant following G418 treatment. Taken together, the results of the present study suggest that the type of premature stop codon and the context of the nucleotide immediately following at the +4 position, may determine the pharmacological rescue efficiency of the HERG gene.

\section{Introduction}

Long QT syndrome (LQTs) is an inherited cardiac arrhythmia syndrome that is associated with syncope and sudden death (1-3). It has been shown that mutations exist in 15 genes encoding either ion channels or structural proteins associated with LQTs (4-7). A previous study revealed that nonsense mutations, which introduce premature termination codons (PTCs) into the gene of interest, account for $\sim 10 \%$ of all mutations (8). When a nonsense mutation occurs in the human etheragogorelated (HERG) gene, which encodes the $\alpha$-subunit of the rapid delayed rectifier potassium current channel (IKr), it may lead to type 2 LQTs.

Aminoglycosides have been demonstrated to have the ability of read-through on nonsense mutations to produce full-length functional proteins in genetic disease models (9-14). Howard et al (11) showed for the first time that G418 suppressed PTCs to restore the function of mutant proteins in 
transiently expressed cDNAs that contained mutations in the cystic fibrosis transmembrane conductance regulator gene in mammalian cells. Our published previously study also demonstrated that G418 and gentamicin partially restored the HERG protein in a dosedependent manner in 293 cells expressing R1014X mutant (15).

The importance of the local sequence context in determining how efficiently aminoglycosides rescue nonsense mutations has been established previously in disease models (16-18). It appears that different stop codons facilitate the termination process with differing efficiencies. The efficiency with which termination may be suppressed can also be influenced by the local sequence context in the immediate vicinity of the stop codons, and for the majority of the disease models, the strongest bias has been found at the position of the base immediately following the stop codon (i.e., the +4 nucleotide). However, at present, how the sequence context may influence the efficiency of aminoglycosides in terms of rescuing HERG nonsense mutants in mammalian cells remains to be fully elucidated. In an attempt to answer this question, the present study aimed to examine the susceptibility of different termination codons, and the local nucleotide sequence surrounding them, in the process of rescuing HERG nonsense mutants by aminoglycosides in 293 cells.

\section{Materials and methods}

HERG mutant cDNA constructs. Wildtype (WT) HERG cDNA cloned into the pcDNA3.1 vector was provided by Dr Zhengfeng Zhou (Oregon Health and Science University, Portland, OR, USA). The HERG nonsense mutations R1014X and W927X, containing different stop codons and +4 nucleotides, were generated in the pcDNA3.1 WT HERG plasmid using a polymerase chain reaction (PCR) based mutagenesis method. PCR was performed as follows: $95^{\circ} \mathrm{C}$ for $2 \mathrm{~min}, 94^{\circ} \mathrm{C}$ for $20 \mathrm{sec}, 55^{\circ} \mathrm{C}$ for $10 \mathrm{sec}, 68^{\circ} \mathrm{C}$ for $2.5 \mathrm{~min}$ for $18 \mathrm{cycles}$, $68^{\circ} \mathrm{C}$ for $5 \mathrm{~min}$. Briefly, the plasmid containing WT HERG cDNA was purified using Endo-Free Plasmid Purification kit (Qiagen, Inc.) according to the manufacture's protocol, and used as the PCR template. The primers with the different mutation sites were added (sequences of primers are shown in Table I), respectively. Amplification was performed using Fast Alteration DNA Polymerase, and a mutated plasmid with a gap was obtained. The methylated template plasmid was digested with DpnI, leaving the mutant plasmid only. Following transfer to the recipient Escherichia coli bacteria, the gap in the mutant plasmid was repaired. The mutant plasmid was obtained and the sequences of the mutants were subsequently verified using ABI PRISM 377 automated sequencer.

Cell culture and transfection. The 293 cells (American Type Culture Collection) were grown in Dulbecco's modified Eagle's medium (DMEM; Gibco; Thermo Fisher Scientific, Inc.) supplemented with $10 \%$ fetal bovine serum (Gibco; Thermo Fisher Scientific, Inc.), $1 \%$ glutamine, $100 \mathrm{IU} / \mathrm{ml}$ penicillin and $100 \mu \mathrm{g} / \mathrm{ml}$ streptomycin at $37^{\circ} \mathrm{C}$ in an incubator in a humidified atmosphere of $5 \% \mathrm{CO}_{2} / 95 \%$ air. Transfections were performed with Effectene ${ }^{\circledR}$ transfection reagent (Qiagen, Inc.) according to the manufacturer's protocol. The 293 cells were transiently transfected either with $0.4 \mu \mathrm{g}$ WT or an equal quantity of mutant cDNA. The green fluorescent protein (GFP) gene $(0.2 \mu \mathrm{g})$ was cotransfected as an indicator during patch-clamp recording. The transfection efficiency was $\sim 65 \%$ (Fig. 1A-C). Pharmacological rescue was examined $24 \mathrm{~h}$ after adding G418 (400 $\mu \mathrm{g} / \mathrm{ml})$ into DMEM.

Immunofluorescence and confocal imaging. An immunofluorescence method was used to observe the protein expression of HERG prior to and following drug treatment. The 293 cells were washed three times with PBS and were subsequently soaked in $4 \%$ paraformaldehyde for $30 \mathrm{~min}$ at room temperature, followed by washing with PBS a further three times. Following permeabilization with $0.5 \%$ Triton $\mathrm{X}-100$ for $20 \mathrm{~min}$, the cells were incubated in antisera against the $\mathrm{N}$ - or C-terminal antibody of HERG protein (Alomone, cat. nos. APC-062 and APC-109, respectively; 1:400 dilution), initially at $37^{\circ} \mathrm{C}$ for $1 \mathrm{~h}$, and then at $4^{\circ} \mathrm{C}$ overnight. Subsequently, the cells were washed three times in PBS and incubated with antirabbit $\mathrm{Cy} 3$-conjugated secondary antibody (Beyotime Institute of Biotechnology, $\mathrm{P} 0183 ; 1: 500$ dilution) at $37^{\circ} \mathrm{C}$ for $30 \mathrm{~min}$, followed by three washes with PBS. Finally, cells were incubated with DAPI (Haoxin Biotech, Inc. or Beyotime Institute of Biotechnology) for $5 \mathrm{~min}$ and then were washed three times in PBS. Confocal images were captured using a confocal laser scanning microscope (Zeiss LSM510; Zeiss $\mathrm{GmbH}$, Jena, Germany) and analyzed using Volocity ${ }^{\circledR}$ Demo software (version 6.0; PerkinElmer, Inc.).

Patch-clamp recording. The wholecell patch-clamp method was used in experiments to record the HERG current. The bathing solution contained the following: $136 \mathrm{mM} \mathrm{NaCl}$, $5.4 \mathrm{mM} \mathrm{KCl}, 0.33 \mathrm{mM} \mathrm{NaH}{ }_{2} \mathrm{PO}_{4} .2 \mathrm{H}_{2} \mathrm{O}, 1.8 \mathrm{mM} \mathrm{CaCl}$, $1 \mathrm{mM} \mathrm{MgCl} 2 \cdot 6 \mathrm{H}_{2} \mathrm{O}, 10 \mathrm{mM}$ glucose and $10 \mathrm{mM}$ HEPES. The internal pipette solution contained the following: $140 \mathrm{mM}$ $\mathrm{KCl}, 0.5 \mathrm{mM} \mathrm{MgCl} \mathrm{m}_{2} \cdot 6 \mathrm{H}_{2} \mathrm{O}, 5 \mathrm{mM}$ HEPES, 2 mM EGTA and $4 \mathrm{mM} \mathrm{K}_{2}$ ATP. The HERG current was activated by a depolarizing pulse (for $4 \mathrm{sec}$ ) to $50 \mathrm{mV}$ from the holding potential of $-80 \mathrm{mV}$. The tail current of the HERG channel was recorded following repolarization to $-50 \mathrm{mV}$ for $4 \mathrm{sec}$. All patch-clamp experiments were performed at room temperature $\left(22 \pm 1^{\circ} \mathrm{C}\right)$.

Statistical analysis. All results are expressed as the mean \pm SEM, and the data were analyzed using SPSS software, version 17.0 (SPSS, Inc., Chicago, IL, USA). For statistical analyses, Student's t-test was performed for comparisons between two data groups. For comparisons of data among more than two groups, one-way analysis of variance was used, followed by Bonferroni post-hoc test. $\mathrm{P}<0.05$ was considered to indicate a statistically significant difference.

\section{Results}

Construction of HERG nonsense mutants containing different stop codons and different downstream nucleotides. The present study aimed to investigate whether different types of stop codon and the downstream nucleotide context, can influence the ability of aminoglycosides to efficiently suppress PTCs. To accomplish this, R1014X and W927X mutants, containing TGA, TAG and TAA, were constructed using a sitedirected mutagenesis technique. Based on the R1014X-TGAT mutant, 
Table I. Primer sequences of the site-directed mutants.

\begin{tabular}{ll} 
Site-directed mutant & \multicolumn{1}{c}{ Primer sequence } \\
\hline R1014X-TGAT & F: 5'-GTACCAGGAGCTCCCTTGATGCCCCGCCCCC-3' \\
R1014X-TAAT & R: 5'-GGGGGCGGGGCATCAAGGGAGCTCCTGGTAC-3' \\
R1014X-TAGT & F: 5'-GTACCAGGAGCTCCCTTAATGCCCCGCCCCC-3' \\
& R: 5'-GGGGGCGGGGCATTAAGGGAGCTCCTGGTAC-3' \\
R1014X-TGAC & F: 5'-GTACCAGGAGCTCCCTTAGTGCCCCGCCCCC-3' \\
& R: 5'-GGGGGCGGGGCACTAAGGGAGCTCCTGGTAC-3' \\
R1014X-TGAG & F: 5'-GTACCAGGAGCTCCCTTGACGCCCCGCCCCC-3' \\
& R: 5'-GGGGGCGGGGCGTCAAGGGAGCTCCTGGTAC-3' \\
R1014X-TGAA & F: 5'-GTACCAGGAGCTCCCTTGAGGCCCCGCCCCC-3' \\
W927X-TGAG & R: 5'-GGGGGCGGGGCCTCAAGGGAGCTCCTGGTAC-3' \\
& F: 5'-GTACCAGGAGCTCCCTTGAAGCCCCGCCCCC-3' \\
W927X-TAAG & R: 5'-GGGGGCGGGGCTTCAAGGGAGCTCCTGGTAC-3' \\
& F: 5'-CGGGGGGGCCGTGAGGGGAGAGCCCG-3' \\
W927X-TAGG & R: 5'-CGGGCTCTCCCCTCACGGCCCCCCCG-3' \\
& F: 5'-CGGGGGGGCCGTAAGGGGAGAGCCCG-3' \\
& R: 5'-CGGGCTCTCCCCTTACGGCCCCCCCG-3' \\
& F: 5'-CGGGGGGGCCGTAGGGGGAGAGCCCG-3' \\
& R: 5'-CGGGCTCTCCCCCTACGGCCCCCCCG-3'
\end{tabular}

F, forward; R, reverse.
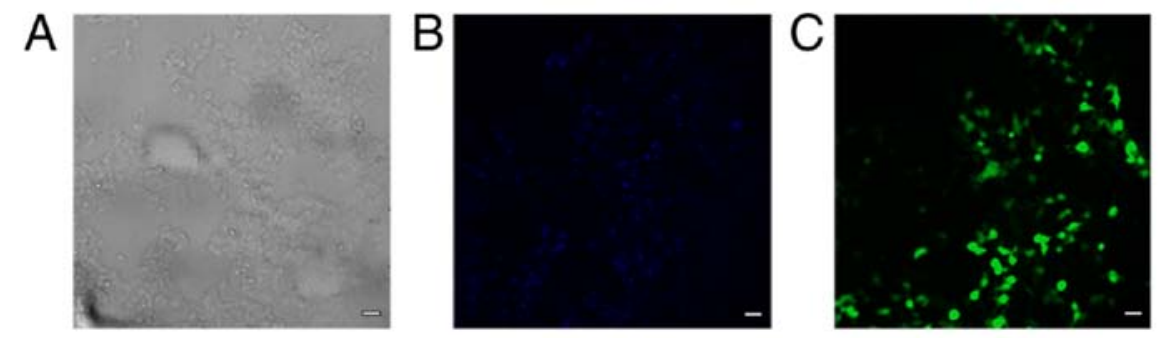

Figure 1. Transfection efficiency of 293 cells. (A) Cells under the light microscope. (B) Expression in the nucleus using DAPI. (C) Cells successfully transfected with GFAP and wild-type plasmids. Scale bar, $100 \mu \mathrm{m}$.

R1014X-TGAA, R1014X-TGAC and R1014X-TGAG mutants were also constructed for the present study (Fig. 2A and B).

Effect of PTCs on the G418-mediated suppression of the R1014X and W927X mutants. Using an immunofluorescence method, the protein expression of HERG in 293 cells transfected with the respective R1014X mutants was detected. Protein expression was detected in the control group (transfection reagent only) and the HERG-WT group prior to and following G418 treatment in 293 cells (Fig. 3). No red fluorescence was detected in the control group with or without G418 treatment (Fig. 3A-D). In the WT group, red fluorescence was detected when antibody against the $\mathrm{N}$-terminal (Fig. 3F), or C-terminal (Fig. 3G) were used prior to G418 treatment, but not when no antibody was added (Fig. 3E). Following treatment with G418, red fluorescence was also detected following addition of the anti-C-terminal antibody (Fig. 3H). An antibody raised against the $\mathrm{N}$-terminal sequence of HERG protein was incubated with the protein prior to the addition of G418. As shown in Fig. 4A-C, red fluorescence was detected in all three groups of cells (i.e., transfected with R1014X-TGA, R1014X-TAA or R1014X-TAG mutant, respectively). By contrast, no red fluorescence was detected when an antibody raised against the C-terminus of the HERG protein was used (Fig. 4D-F). However, following treatment with G418 $(400 \mu \mathrm{g} / \mathrm{ml})$, red fluorescence was observed in each group following incubation with the anti-C-terminal antibody (Fig. 4G-I).

Ionic currents were also recorded for the 293 cells transiently transfected with WT cDNA and R1014X mutant containing the different stop codons (Fig. 5A). The mean peak tail current densities of R1014X-TGA $(4.82 \pm 0.42 \mathrm{pA} / \mathrm{pF}$; $\mathrm{n}=8), \mathrm{R} 1014 \mathrm{X}-\mathrm{TAA}(5.31 \pm 0.62 \mathrm{pA} / \mathrm{pF} ; \mathrm{n}=6)$ and R1014X-TAG $(4.51 \pm 0.68 \mathrm{pA} / \mathrm{pF} ; \mathrm{n}=6)$ were all lower compared with those of the WT channel $(46.69 \pm 3.74 \mathrm{pA} / \mathrm{pF} ; \mathrm{n}=6 ; \mathrm{P}<0.05)$. However, no significant differences were observed among these three mutants. An increase in the peak tail current was only identified with the R1014X-TGA $(21.64 \pm 1.94 \mathrm{pA} / \mathrm{pF} ; \mathrm{n}=6 ; \mathrm{P}<0.05)$ 


\section{A Nucleotide sequence}

$$
\begin{array}{lllllllll}
-6 & -5 & -4 & -3 & -2 & -1 & \text { stop codon }+4+5+6+7 & +8 & +9
\end{array}
$$

B

R1014X-TGAT
R1014X-TAAT
R1014X-TAGT
R1014X-TGAC
R1014X-TGAG
R1014X-TGAA

W927X-TGAG W927X-TAGG W927X-TAAG ggc cgc cag tac cag gag ctc cct cga tgc ccc gcc ccc acc

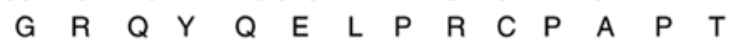

ggc cgc cag tac cag gag ctc cct tga tgc ccc gcc ccc acc ggc cgc cag tac cag gag ctc cct taa tgc ccc gcc ccc acc ggc cgc cag tac cag gag ctc cct tag tgc ccc gcc ccc acc ggc cgc cag tac cag gag ctc cct tga $\mathrm{cgc}$ ccc gcc ccc acc ggc cgc cag tac cag gag ctc cct tga g gc ccc gcc ccc acc ggc cgc cag tac cag gag ctc cct tga a gc ccc gcc ccc acc

agc cgg ggc cgg ccg ggg ggg ccg tgg ggg ggg agc ccg tcc

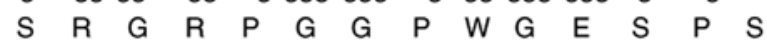
agc cgg ggc cgg ccg ggg ggg ccg tga ggg ggg agc ccg tcc agc cgg ggc cgg ccg ggg ggg ccg tag ggg ggg agc ccg tcc agc cgg ggc cgg ccg ggg ggg ccg taa ggg ggg agc ccg tcc

Figure 2. R1014X and W927X mutants. (A) Nucleotide sequence surrounding the stop codon. (B) R1014X and W927X mutants containing different stop codons (TGA, TAA and TAG within boxes) and different +4 nucleotides immediately downstream of the stop codon, TGA, are shown. The single-letter abbreviations of the amino acids are as follows: A, alanine; R, arginine; C, cysteine; Q, glutamine; E, glutamic acid; G, glycine; L, leucine; P, proline; S, serine; T, threonine; W, tryptophan; Y, tyrosine.
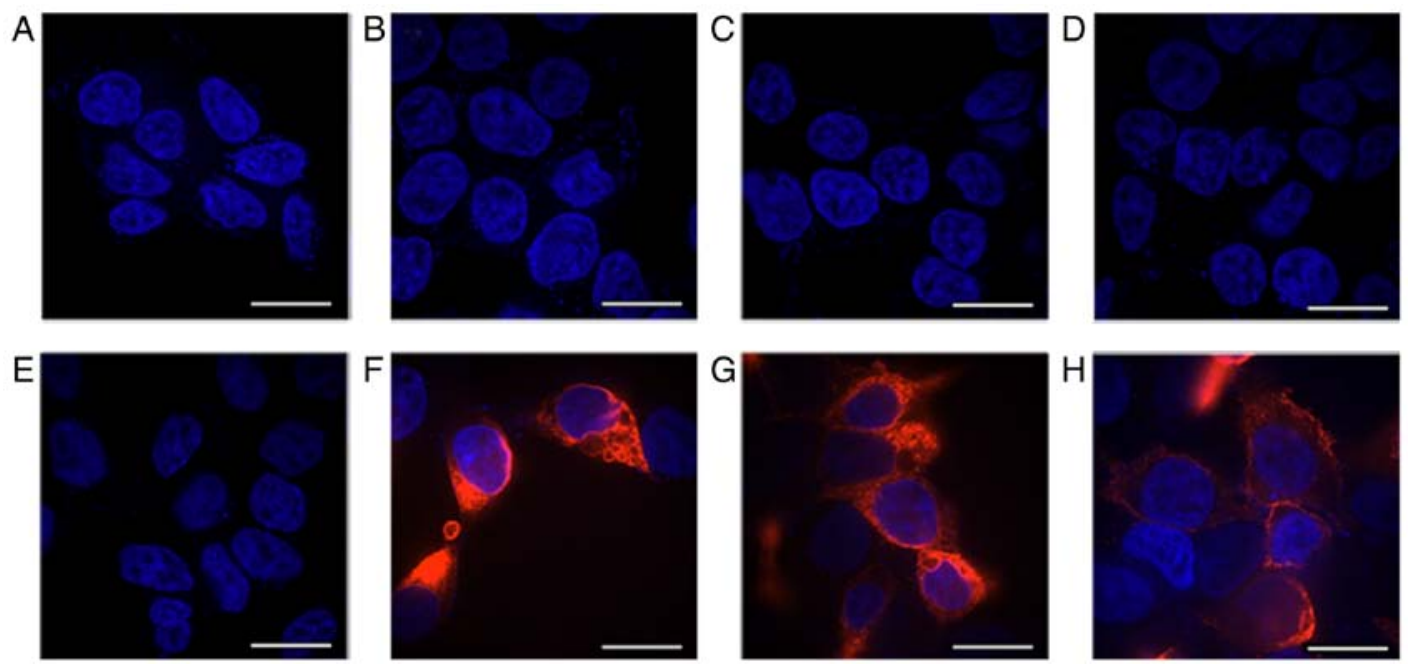

Figure 3. Protein expression of HERG in the control and HERG-WT groups before and after G418 treatment in 293 cells. Control group (transfection reagent only): (A) no antibody added before G418 treatment; (B) antibody against the N-terminus of HERG protein added before G418; (C) anti-C-terminal antibody added before G418 treatment; (D) anti-C-terminal antibody added following treatment with G418. HERGWT group: (E) no antibody added before G418; (F) antibody against the N-terminus of HERG protein added before G418; (G) anti-C-terminal antibody added before G418 treatment; (H) anti-C-terminal antibody following treatment with G418. Red fluorescence represents the expression of HERG protein. Scale bar, $100 \mu \mathrm{m}$. HERG, human ether-a-go-go-related; WT, wild-type.

and R1014X-TAA $(23.19 \pm 3.27 \mathrm{pA} / \mathrm{pF} ; \mathrm{n}=6 ; \mathrm{P}<0.05)$ mutants, and not with the R1014X-TAG mutant, subsequent to the rescuing effect of G418 (Fig. 5B).

To further validate these phenomena, the same treatment was applied to W927X mutants, which featured the PTCs of TGA, TAA and TAG, respectively. The immunofluorescence results (Fig. 6A-I) revealed that red fluorescence was detected in all three mutants bearing the different types of PTC following the administration of G418 using the antibody raised against the C-terminus (Fig. 6). The patchclamp results suggested that the mean peak tail electric current density was increased in 293 cells transfected with W927X-TGA, W927X-TAA and W927X-TAG following treatment with G418. However, the increase in current density with W927X-TAG was smaller compared with that with the other two mutants (Fig. 7A and B; $\mathrm{P}<0.05)$.

Role of the tetranucleotide termination signal on the rescuing effect of $G 418$. To observe the impact of the local sequence context surrounding the PTC, the predominant focus of the present study was on the +4 nucleotide, and based on the R1014X-TGAT mutant, R1014X-TGAC, R1014X-TGAG and R1014X-TGAA mutants were constructed. Prior to the addition of G418, the N-terminal antibody was used to observe the protein expression of HERG among the groups. Using the immunofluorescence method, red fluorescent protein was detected in each group (Fig. 8A-C). However, when the Cterminal antibody was used, no red fluorescence was detected (Fig. 8D-F). The functional expression of 
A

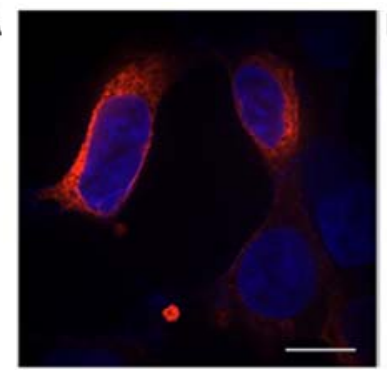

D

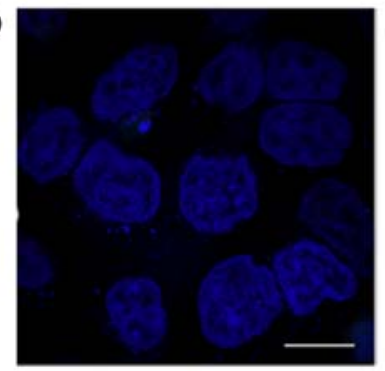

G

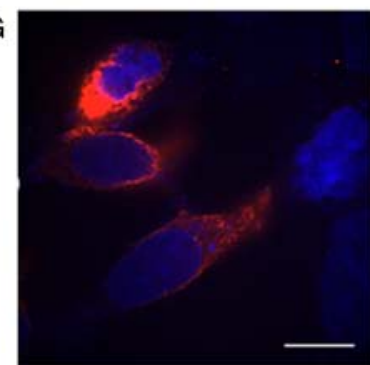

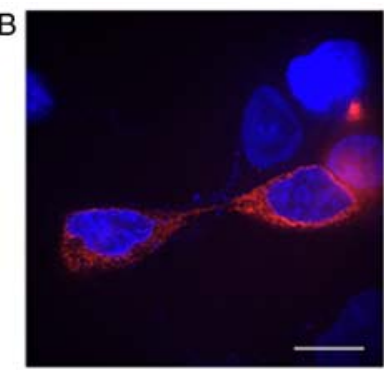
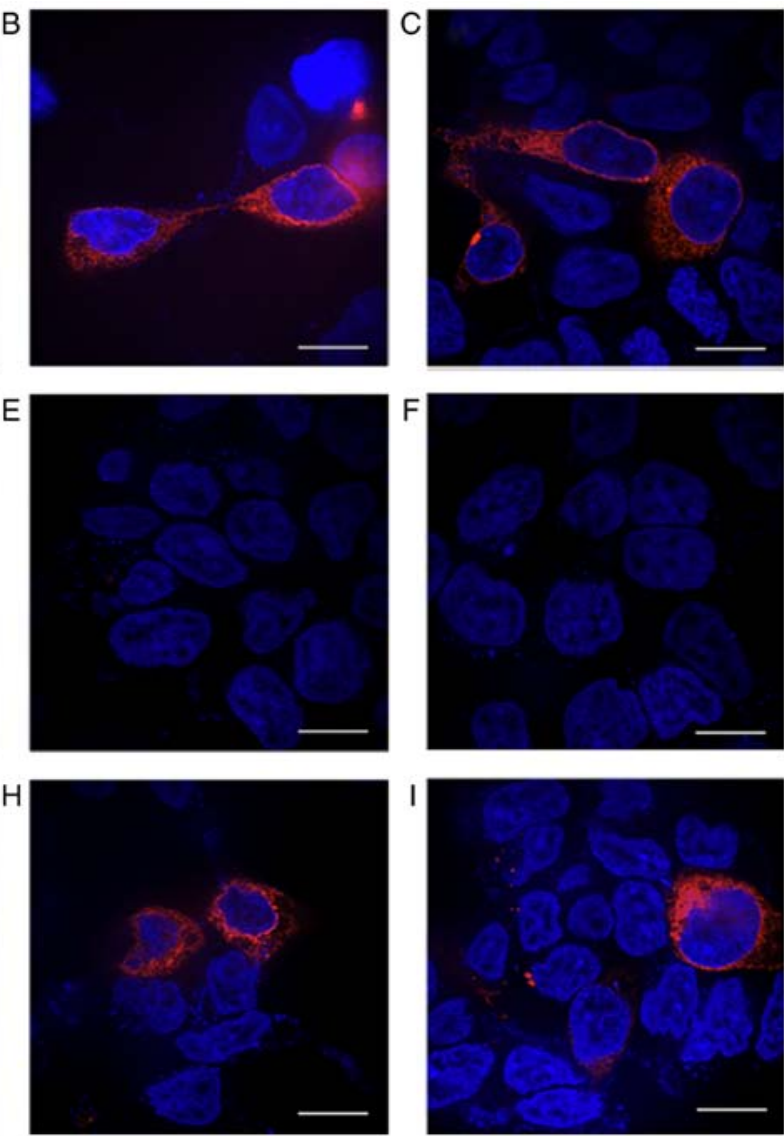

Figure 4. Protein expression of the HERG-R1014X mutant with stop codon TGA, TAA or TAG prior to and following G418 treatment in 293 cells. Antibody raised against the $\mathrm{N}$-terminus of the HERG protein was used before G418 was added in the (A) TGA, (B) TAA and (C) TAG groups. Antibody against the C-terminus of the HERG protein was used before G418 was added to the (D) TGA, (E) TAA and (F) TAG groups. Following treatment with G418, anti-C-erminal antibody was used in the (G) TGA, (H) TAA and (I) TAG groups. Red fluorescence represents the expression of HERG protein. Scale bar, $100 \mu \mathrm{m}$. HERG, human ether-a-go-go-related.

A

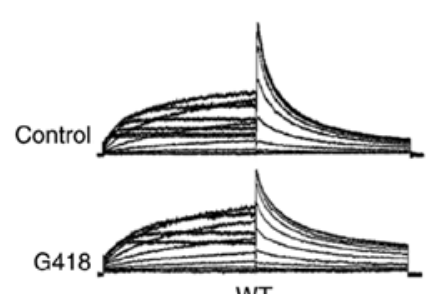

WT

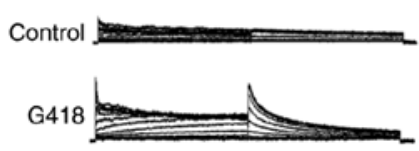

R1014X-TAA
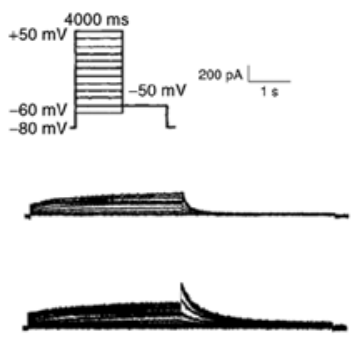

R1014X-TGA

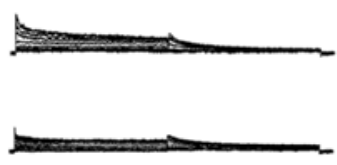

R1014X-TAG
B

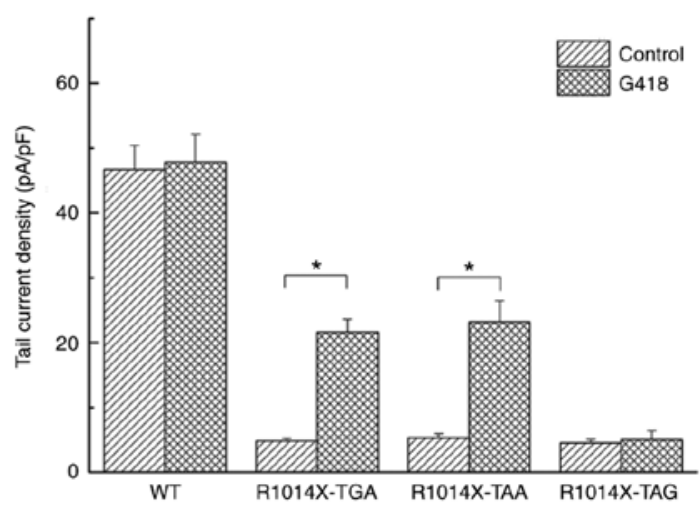

Figure 5. Ionic currents recorded for the WT and R1014X mutant HERG channels before and after G418 treatment in transfected 293 cells. (A) Representative families of current traces recorded from cells transfected with WT or the three mutant HERG cDNAs are shown. (B) Peak tail currents recorded for the cells transfected with WT or R1014X mutant proteins with different stop codons. * $\mathrm{P}<0.05$. HERG, human ether-a-go-go-related; WT, wild-type.

HERG protein in each group without drug treatment was subsequently tested by patch-clamp recording. On comparing the peak tail current densities, no significant differences were identified among the groups $(5.58 \pm 1.33,5.16 \pm 0.99$ and $3.80 \pm 1.08 \mathrm{pA} / \mathrm{pF}$ for the R1014X-TGAC, R1014X-TGAG and R1014X-TGAA mutants, respectively; Fig. 9A and $\mathrm{B})$.
Following G418 treatment, red fluorescence was detected in all groups using the C-terminal antibody (Fig. 8G-I). For the group transfected with R1014X-TGAT, the peak tail current density was increased in the cells treated with G418 $(22.96 \pm 1.75 \mathrm{pA} / \mathrm{pF} ; \mathrm{n}=5 ; \mathrm{P}<0.05)$ compared with that in the untreated cells $(4.54 \pm 0.51 \mathrm{pA} / \mathrm{pF} ; \mathrm{n}=6)$, although 

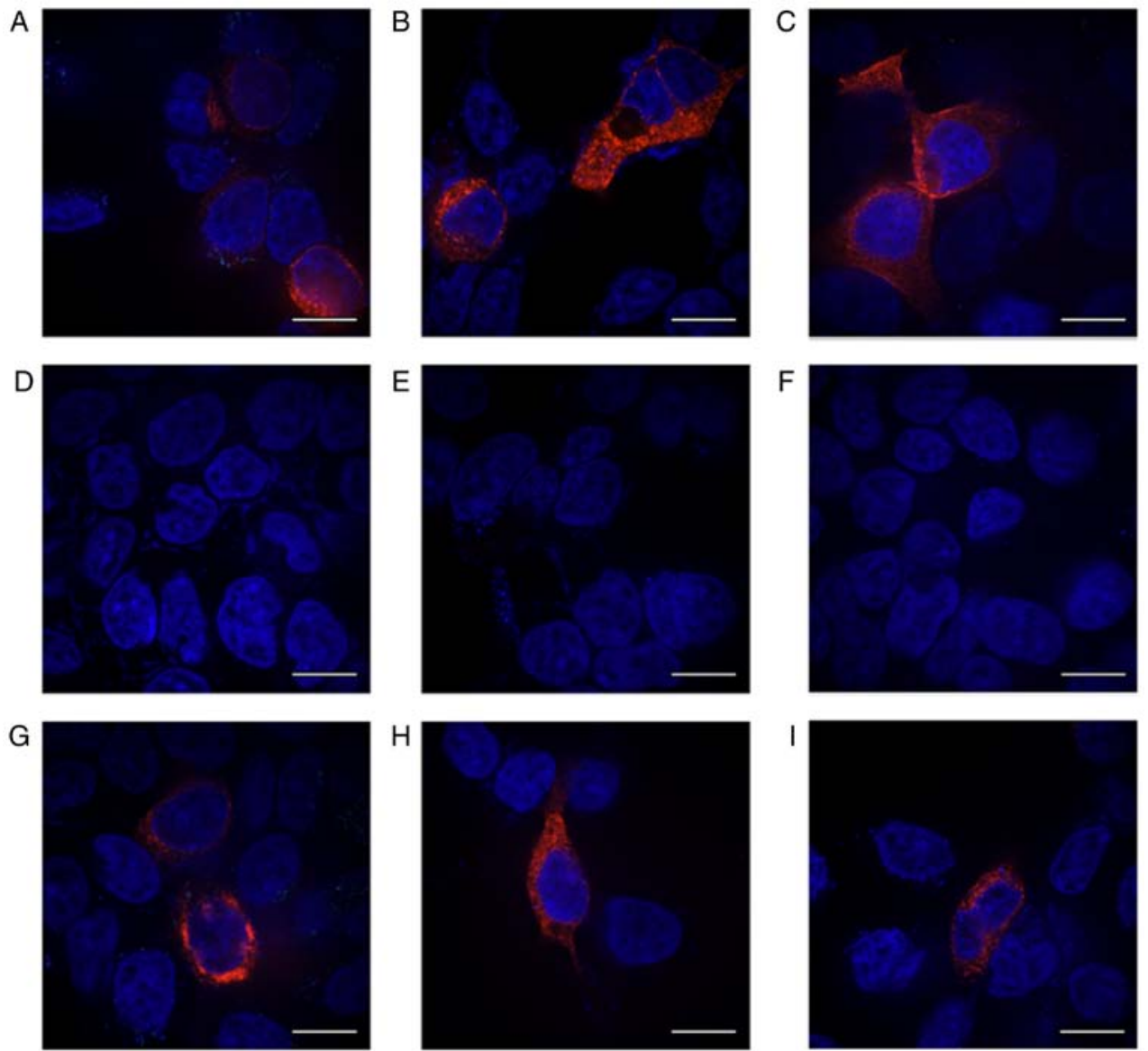

Figure 6. Protein expression levels in cells transfected with HERG-W927X mutant with stop codon TGA, TAA, or TAG, respectively, before and after G418 treatment. (A) TGA, (B) TAA and (C) TAG groups in which antibody raised against the N-terminus of the HERG protein was used before G418. (D) TGA, (E) TAA and (F) TAG groups in which antibody against the C-terminus of the HERG protein was used before G418. (G) TGA, (H) TAA and (I) TAG groups in which anti-C-terminal antibody was used following treatment with G418. Red fluorescence represents the expression of the HERG protein. Scale bar, $100 \mu \mathrm{m}$. HERG, human ether-a-go-go-related.

this was still lower compared with that of the WT channel $(47.83 \pm 4.31 \mathrm{pA} / \mathrm{pF} ; \mathrm{n}=5)$. In the other groups transfected with R1014X-TGAC, R1014X-TGAG or R1014X-TGAA, the tail current density did not alter significantly upon treatment with G418 $(5.58 \pm 1.33$ vs. $5.45 \pm 1.84 \mathrm{pA} / \mathrm{pF}$, $5.16 \pm 0.99 \mathrm{vs} .5 .65 \pm 1.50 \mathrm{pA} / \mathrm{pFand} 3.80 \pm 1.08 \mathrm{vs} .5 .84 \pm 1.46 \mathrm{pA} / \mathrm{pF}$, respectively; all $\mathrm{P}>0.05$; Fig. $9 \mathrm{~A}$ and $\mathrm{B}$ ).

\section{Discussion}

In the present study, to investigate how stop codons and the +4 nucleotide may influence the efficiency of G418 in rescuing nonsense mutations of the HERG gene, the possible impacting factors were examined, such as the type of PTC and the nucleotide sequences immediately downstream of them. It was revealed that the stop codons TGA and TAA were rescued more easily by the aminoglycoside compared with TAG. In addition, the +4 nucleotide influenced the function of HERG protein following the rescuing effect of the aminoglycoside. This is the first time, to the best of our knowledge, that the effect of the intrinsic sequence on the nonsense mutation rescue of the HERG gene has been observed. The HERG gene was a particularly good candidate for PTC suppression, as it has a higher frequency of nonsense mutations than other genes associated with arrhythmia and sudden death, the majority of which are inactivated by missense mutations.

The read-through effect on nonsense mutations to produce full-length functional proteins has been demonstrated for aminoglycosides in numerous genetic disease models, including Duchenne muscular dystrophy (DMD) and ocular genetic diseases $(19,20)$. In our previous study, the expression of WT and R1014X mutant in 293 cells was confirmed by western blotting and PCR following the transfection of corresponding plasmids, respectively. It was also demonstrated that G418 and gentamicin partially restored the HERG-like currents in 293 cells expressing W927X and R1014X mutants (15). It has also been reported that the type of stop codon can influence the rescuing efficiency of an aminoglycoside. Gentamicin and G418 enabled aminoacyl-tRNA to read through UGA more easily than other PTCs in disease models associated with APC or p53 gene nonsense mutations $(18,21)$. In clinical trials associated with DMD, upon administration of gentamicin, the level of the myotubes was upregulated more markedly in patients with the PTC of UGA, followed by UAA and UAG (22). In the present study, the results confirmed that G418 suppressed UGA and UAA, and the IKr current was restored more readily for these PTCs than for UAG in HERG nonsense mutations.

The context of the stop codon and its surrounding sequences influence the efficiency of translation termination, 
A

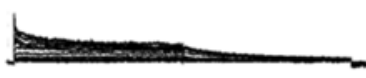

W927X-TGA

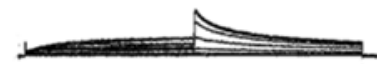

W927X-TGA+G418

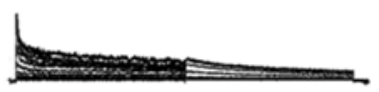

W927X-TAA

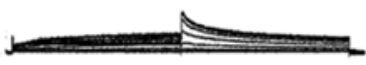

W927X-TAA+G418

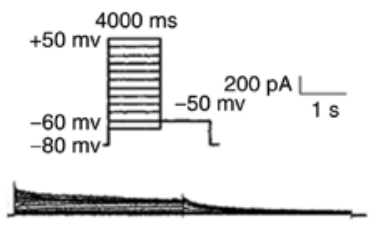

W927X-TAG

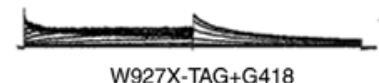

W927X-TAG+G418

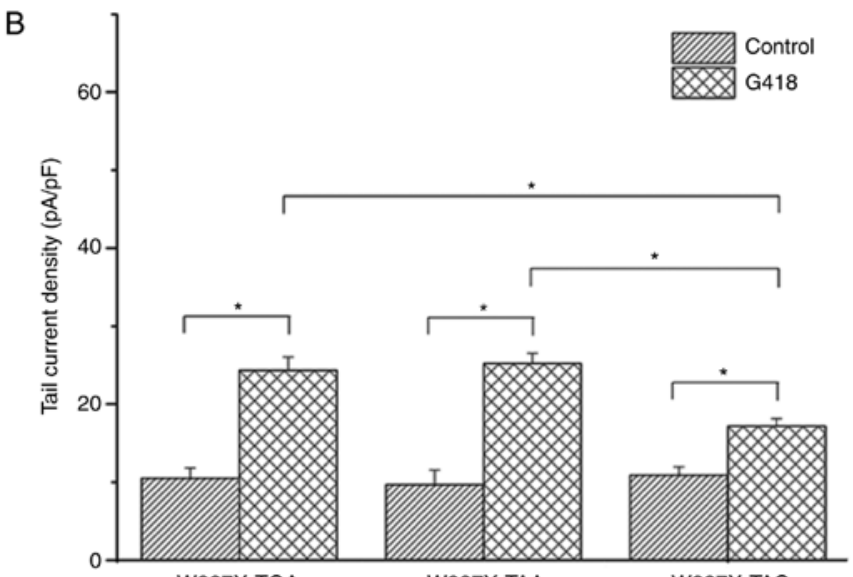

Figure 7. Ionic current recorded from W927X mutant HERG channels before and after G418 treatment in transfected 293 cells. (A) Representative families of current traces recorded from cells transfected with WT or the three mutant HERG cDNAs. (B) Peak tail currents from cells transfected with the W927X mutants featuring the different stop codons. ${ }^{*} \mathrm{P}<0.05$. HERG, human ether-a-go-go-related; WT, wild-type.
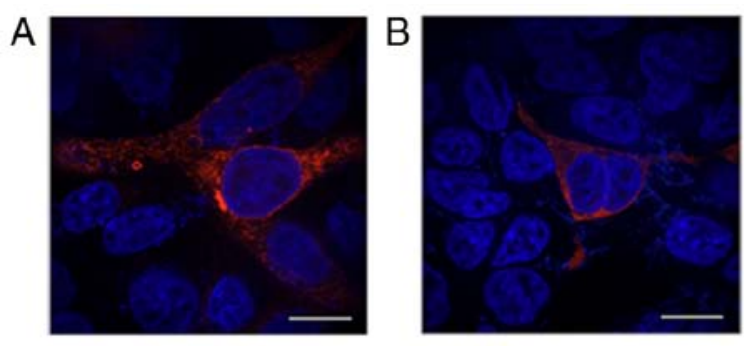

E
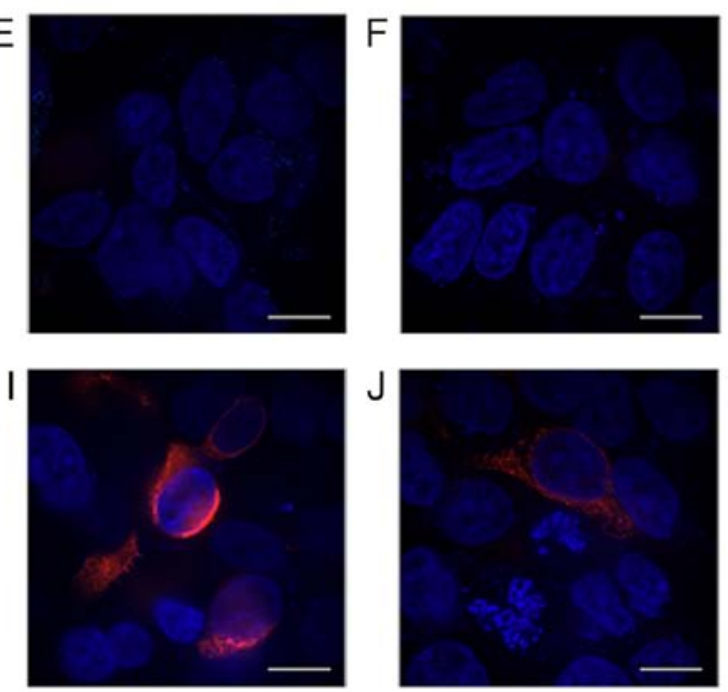
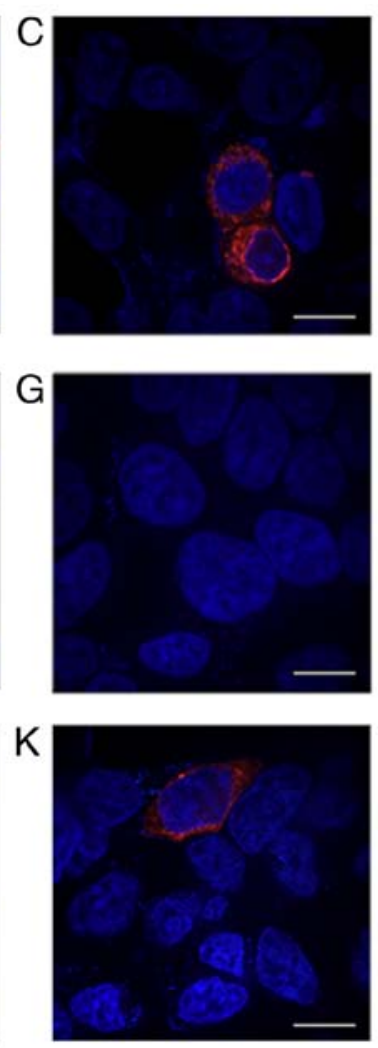
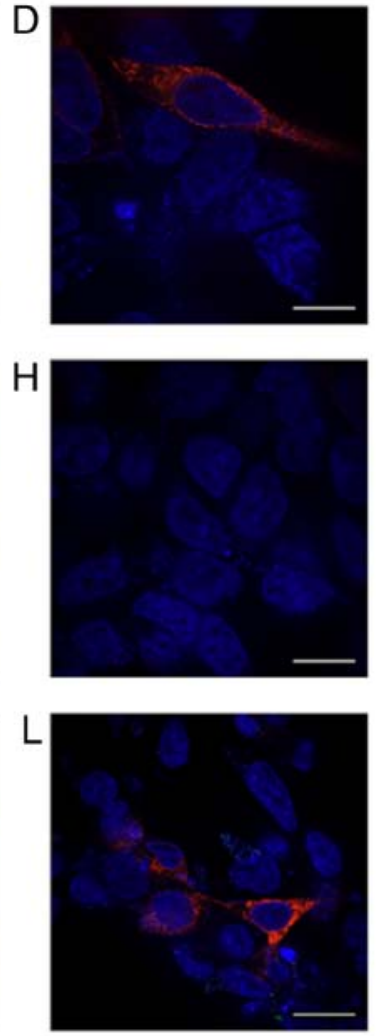

Figure 8. Protein expression in cells transfected with HERG-R1014X mutant containing different +4 nucleotides downstream of the stop codon TGA before and after G418 treatment. Antibody against the N-terminus of the protein was used before G418 was added to mutants with the +4 nucleotides (A) T, (B) G, (C) A and (D) C, respectively. Antibody against the C-terminus of the protein was used before mutants with the +4 nucleotides (E) T, (F) G, (G) A and (H) C were treated with G418. (I-L) Following treatment with G418, anti-C-terminal antibody was used for the (I) T, (J) G, (K) A and (L) C mutants. Red fluorescence represents expression of the HERG protein. Scale bar, $100 \mu \mathrm{m}$. HERG, human ether-a-go-go-related. 
A

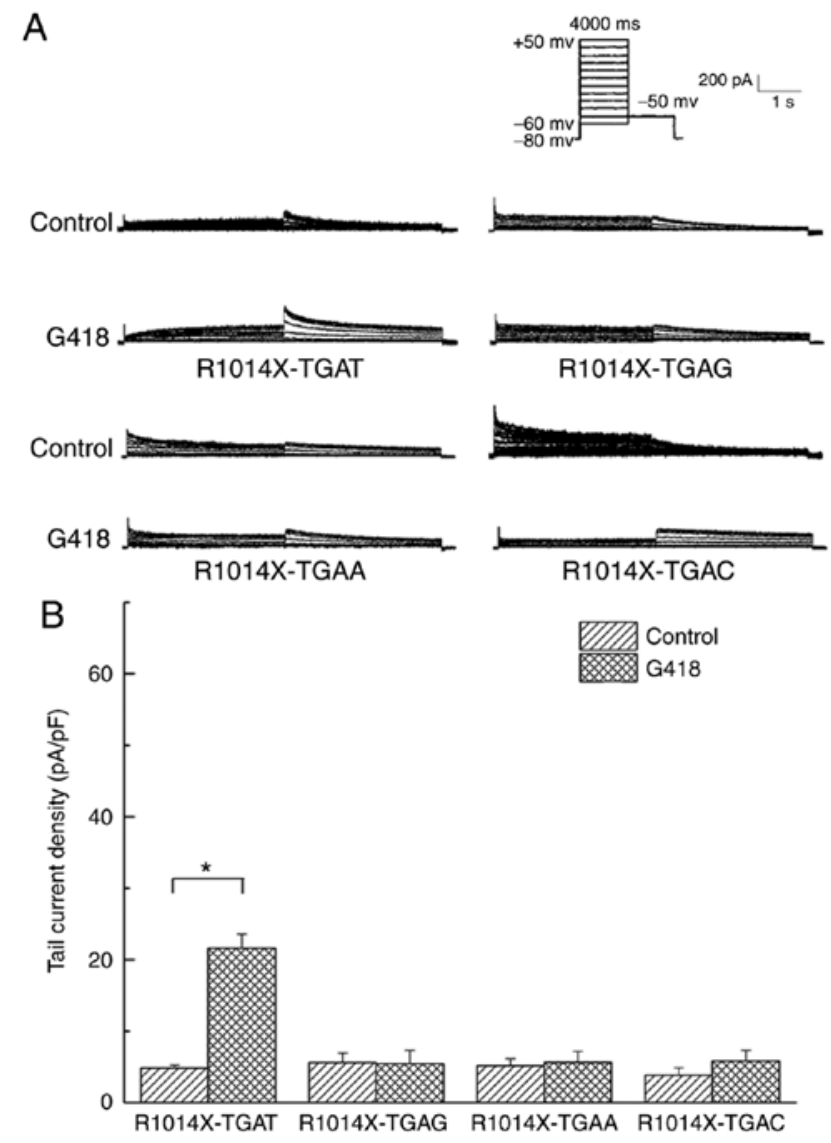

Figure 9. Ionic current recorded from the R1014X mutant HERG channels before and after G418 treatment of the transfected 293 cells. (A) Representative families of current traces recorded from cells transfected with the four mutant HERG cDNAs. (B) Peak tail currents from cells transfected with the R1014X mutants with the different +4 nucleotides downstream of the stop codon TGA. ${ }^{*} \mathrm{P}<0.05$. HERG, human ether-a-go-go-related; WT, wild-type.

as has been reported in previous studies $(23,24)$. However, the mechanism remains unclear. Aminoglycoside antibiotics can exert their effect by binding to the A site of ribosomal rRNA via hydrogen bonds, thus altering the conformation of ribosomal rRNA, mRNA and aminoacyl-tRNA. This effect reduces the fidelity of the translation process, causing similar aminoacyl-tRNAs to enter the A site. When an amino is added to the stop codon site, the read-through effect occurs (25).

It has been reported that, when WT AUGG was mutated to AUAG, AUGA and AUAA in nonsense mutation models, the ability to bind to ribosomal and aminoacyl transfer RNA was $0.1,3.2$ and 0.3 times higher compared with AUGG, respectively $(25,26)$. This means that, among the three stop codons, UGA was more likely to bind to the aminoacyl-tRNA in the ribosome, inhibiting the termination of translation and causing the protein to continue to synthesize. Therefore, G418 may induce more efficient translational read-through and produce sufficient full-length protein in the nonsense mutation with stop codon UGA or UAA. Additional investigations are required to obtain further understanding of the termination of translation at normal and premature stop codons. It is important to characterize the identity of the amino acid inserted by the treatment of G418 in HERG nonsense mutations with different stop codons.
Nonsense-mediated decay (NMD) is an RNA quality control mechanism that selectively degrades mRNA harboring PTCs. NMD eliminates abnormal mRNA transcripts harboring PTCs, thereby preventing the production of truncated proteins that often have dominant-negative effects (27). However, the role of NMD in the majority of eukaryotic cells requires at least one intron downstream of the PTCs and the corresponding splicing process. Pre-mRNA splicing results in the deposition of a multi-protein complex, known as the exon-junction-complex (EJC), 20-24 nt upstream of each exon-exon junction. The EJCs are displaced by the ribosome during the pioneer round of translation. If translation terminates at a PTC that is located 50-55 nt upstream of an exon-exon junction, the downstream EJC serves as a binding platform for NMD factors that trigger NMD. If no intron exists, the mRNA can escape NMD. In the present study, cDNA of the HERG gene containing exons was used to rule out the interference of NMD on the experimental results.

The efficiency of translation termination is also affected by upstream and downstream sequences around the PTC, particularly by the +4 nucleotide $(28,29)$. When an adenine nucleotide is located at this position, the highest rescuing efficiency may be detected, followed by a pyrimidine nucleotide at the same position (30-32). Therefore, it was hypothesized that the local sequence context featuring the +4 nucleotide is also involved in the process of translation termination, in addition to the PTC. Previous studies on DMD and other disease models have confirmed the important influence of the +4 nucleotide $(33,34)$. In a QX $(\mathrm{N})$ expression system, it was revealed that the stop codon had a close association with the +4 nucleotide (35). Gentamicin and other aminoglycosides were observed to have different rescuing efficiencies with different types of nucleotide at the +4 position. When the stop codon was TGA, the effectiveness of the +4 nucleotide was in the order of $\mathrm{C}>\mathrm{T}, \mathrm{A}>\mathrm{G}$ (35).

The results of the present study suggested that the +4 nucleotide downstream of the stop codon had a significant impact on the functional expression of HERG protein following the rescue of readthrough by the drug. When the original base $\mathrm{T}$ was mutated to $\mathrm{C}, \mathrm{G}$ or $\mathrm{A}$, respectively, red fluorescence remained apparent following the addition of an antibody against the C-terminus of HERG following G418 treatment, indicating expression of the intact HERG protein. However, the peak tail current did not alter significantly following the administration of G418. Amino acid sequence analysis predicted that nucleotide mutation results in the original-site cysteine (corresponding to the nucleotide sequence, tgc) being mutated to arginine (corresponding to nucleotide sequence, $\mathrm{cgc}$ ), serine (corresponding to nucleotide sequence, agc) or glycine (corresponding to the codon, ggc). Therefore, a mutation at the +4 nucleotide base position altered the amino acid sequence coded by the nucleotides downstream of the stop codon upon intervention with G418. In this manner, no functional integral protein can be produced. Notably, these results differed from previous results obtained in other disease models $(31,34)$. Due to the lack of suitable technology, it is not possible to analyze and predict the amino acid that is inserted into the site of the PTC. However, the results of our previous study demonstrated that certain HERG nonsense mutants, such as R863X, E698X and W412X, positioned close to the Nterminus, were not rescued upon treatment with an aminoglycoside (15); therefore, the amino acid may differ from the 
original amino acid upon rescue with G418. If the mutation is located in the domain of the HERG gene that is most critical for protein structure, the conformation of the HERG protein may be altered upon rescue with an aminoglycoside, leading to its function not being restored. However, further investigations are required in order to elucidate the specific mechanism.

In conclusion, the present study demonstrated that the type of PTC, and the context of the nucleotides downstream of it, may determine the pharmacological rescue efficiency of the HERG gene. TGA and TAA were more easily rescued by the aminoglycoside compared with TAG. Furthermore, the +4 nucleotide was found to influence the function of the HERG protein following the rescuing effect of the aminoglycoside. By identifying the type of stop codon and the following sequence, clinicians can establish patients who are more sensitive to nonsense mutation rescue therapy among those with LQT2 in the future. Pacemaker implantation, which is expensive and traumatic, may be avoided in these individuals. However, these findings highlight the fact that the mechanism of aminoglycosidemediated suppression in mammalian cells remains to be fully elucidated.

In the present study, GFP and the target gene were not placed in the same plasmid. Therefore, it was only possible to demonstrate successful transfection of the target gene through the expression of GFP indirectly. However, by recording the HERG current and using an antibody against the target gene, the expression of the target gene was confirmed. Additionally, transfection of the 293 cells in the experiments was performed with Effectene transfection reagent according to the manufacturer's instructions. After $24 \mathrm{~h}$, Pharmacological rescue was assessed by adding G418 (400 $\mu \mathrm{g} / \mathrm{ml})$ into DMEM for another $24 \mathrm{~h}$. As there was a period of only $48 \mathrm{~h}$ after transfection, it was not possible to examine the stability of HERG protein in different groups. This is an important question and it is sensible to examine the stability of HERG protein with long-tern drug administration in nonsense mutation animal models.

\section{Acknowledgements}

Not applicable.

\section{Funding}

The present study was funded by the National Natural Science Foundation of China (grant no. 81070150) and the Beijing Municipal Natural Science Foundation (grant no. 7192105).

\section{Availability of data and materials}

The datasets used and/or analyzed during the present study are available from the corresponding author upon reasonable request.

\section{Authors' contributions}

HY performed the patch-clamp experiment, analyzed data and was a major contributor in writing the manuscript. YM performed PCR and immunofluorescence experiments and contributed to writing the manuscript. SZ and CT performed cell culture and the transfection experiment. FW, NL and QL analyzed and interpreted the data collected from patch-clamp experiments. YJ contributed to improvements in the manuscript and provided financial support for the study. JP made substantial contributions to conception and design and provided financial support for the study. All authors read and approved the final manuscript.

\section{Ethics approval and consent to participate}

Not applicable.

\section{Patient consent for publication}

Not applicable.

\section{Competing interests}

The authors declare that they have no competing interests.

\section{References}

1. Li G, Shi R, Wu J, Han W, Zhang A, Cheng G, Xue X and Sun C: Association of the hERG mutation with longQT syndrome type 2, syncope and epilepsy. Mol Med Rep 13: 2467-2475, 2016.

2. Czosek RJ, Kaltman JR, Cassedy AE, Shah MJ, Vetter VL, Tanel RE, Wernovksy G, Wray J and Marino BS: Quality of life of pediatric patients with long QT syndrome. Am J Cardiol 117: 605-610, 2016.

3. Kanters JK, Yuan L, Hedley PL, Stoevring B, Jons C, Bloch Thomsen PE, Grunnet M, Christiansen M and Jespersen T: Flecainide provocation reveals concealed brugada syndrome in a long QT syndrome family with a novel L1786Q mutation in SCN5A. Circ J 78: 1136-1143, 2014.

4. Splawski I, Shen J, Timothy KW, Lehmann MH, Priori S, Robinson JL, Moss AJ, Schwartz PJ, Towbin JA, Vincent GM and Keating MT: Spectrum of mutations in long-QT syndrome genes. KVLQT1, HERG, SCN5A, KCNE1, and KCNE2. Circulation 102: 1178-1185, 2000.

5. Marsman RF, Barc J, Beekman L, Alders M, Dooijes D, van den Wijngaard A, Ratbi I, Sefiani A, Bhuiyan ZA, Wilde AA and Bezzina CR: A mutation in CALM1 encoding calmodulin in familial idiopathic ventricular fibrillation in childhood and adolescence. J Am Coll Cardiol 63: 259-266, 2014.

6. Mototani H, Iida A, Nakamura Y and Ikegawa S: Identification of sequence polymorphisms in CALM2 and analysis of association with hip osteoarthritis in a Japanese population. J Bone Miner Metab 28: 547-553, 2010.

7. Ortiz-Bonnin B, Rinne S, Moss R, Streit AK, Scharf M, Richter K, Stöber A, Pfeufer A, Seemann G, Kääb S, et al: Electrophysiological characterization of a large set of novel variants in the SCN5A-gene: Identification of novel LQTS3 and BrS mutations. Pflugers Arch 468: 1375-1387, 2016.

8. Rowe SM and Clancy JP: Pharmaceuticals targeting nonsense mutations in genetic diseases: Progress in development. Biodrugs 23: 165-174, 2009.

9. Floquet C, Hatin I, Rousset JP and Bidou L: Statistical analysis of readthrough levels for nonsense mutations in mammalian cells reveals a major determinant of response to gentamicin. PLoS Genet 8: e1002608, 2012.

10. Brendel C, Belakhov V, Werner H, Wegener E, Gärtner J, Nudelman I, Baasov T and Huppke P: Readthrough of nonsense mutations in Rett syndrome: Evaluation of novel aminoglycosides and generation of a new mouse model. J Mol Med (Berl) 89: 389-398, 2011.

11. Howard M, Frizzell RA and Bedwell DM: Aminoglycoside antibiotics restore CFTR function by overcoming premature stop mutations. Nat Med 2: 467-469, 1996.

12. Baradaran-Heravi A, Balgi AD, Zimmerman C, Choi $\mathrm{K}$, Shidmoossavee FS, Tan JS, Bergeaud C, Krause A, Flibotte S, Shimizu Y, et al: Novel small molecules potentiate premature termination codon readthrough by aminoglycosides. Nucleic Acids Res 44: 6583-6598, 2016. 
13. McHugh DR, Steele MS, Valerio DM, Miron A, Mann RJ, LePage DF, Conlon RA, Cotton CU, Drumm ML and Hodges CA: A G542X cystic fibrosis mouse model for examining nonsense mutation directed therapies. PLoS One 13: e199573, 2018.

14. Bolze F, Mocek S, Zimmermann A and Klingenspor M: Aminoglycosides, but not PTC124 (Ataluren), rescue nonsense mutations in the leptin receptor and in luciferase reporter genes. Sci Rep 7: 1020, 2017.

15. Yu H, Liu X, Huang J, Zhang Y, Hu R and Pu J: Comparison of read-through effects of aminoglycosides and PTC124 on rescuing nonsense mutations of HERG gene associated with long QT syndrome. Int J Mol Med 33: 729-735, 2014.

16. Lee HL and Dougherty JP: Pharmaceutical therapies to recode nonsense mutations in inherited diseases. Pharmacol Ther 136: 227-266, 2012

17. Floquet C, Rousset JP and Bidou L: Readthrough of premature termination codons in the adenomatous polyposis coli gene restores its biological activity in human cancer cells. PLoS One 6: e24125, 2011.

18. Zilberberg A, Lahav L and Rosin-Arbesfeld R: Restoration of APC gene function in colorectal cancer cells by aminoglycosideand macrolide-induced read-through of premature termination codons. Gut 59: 496-507, 2010.

19. Wang $X$ and Gregory-Evans CY: Nonsense suppression therapies in ocular genetic diseases. Cell Mol Life Sci 72: 1931-1938, 2015

20. De Luca A, Nico B, Rolland JF, Cozzoli A, Burdi R, Mangieri D, Giannuzzi V, Liantonio A, Cippone V, De Bellis M, et al: Gentamicin treatment in exercised mdx mice: Identification of dystrophin-sensitive pathways and evaluation of efficacy in work-loaded dystrophic muscle. Neurobiol Dis 32: 243-253, 2008.

21. Floquet C, Deforges J, Rousset JP and Bidou L: Rescue of non-sense mutated p53 tumor suppressor gene by aminoglycosides. Nucleic Acids Res 39: 3350-3362, 2011.

22. Malik V, Rodino-Klapac LR, Viollet L, Wall C, King W, Al-Dahhak R, Lewis S, Shilling CJ, Kota J, Serrano-Munuera C, et al: Gentamicin-induced readthrough of stop codons in Duchenne muscular dystrophy. Ann Neurol 67: 771-780, 2010.

23. Cridge AG, Crowe-McAuliffe C, Mathew SF and Tate WP Eukaryotic translational termination efficiency is influenced by the 3' nucleotides within the ribosomal mRNA channel. Nucleic Acids Res 46: 1927-1944, 2018.

24. Frolova L, Le Goff X, Zhouravleva G, Davydova E, Philippe M and Kisselev L: Eukaryotic polypeptide chain release factor eRF3 is an eRF1- and ribosome-dependent guanosine triphosphatase. RNA 2: 334-341, 1994.
25. Moazed D and Noller HF: Interaction of antibiotics with functional sites in 16S ribosomal RNA. Nature 327: 389-394, 1987.

26. Engelberg-Kulka H: UGA suppression by normal tRNA Trp in Escherichia coli: Codon context effects. Nucleic Acids Res 9: 983-991, 1981.

27. Gong Q, Stump MR and Zhou Z: Position of premature termination codons determines susceptibility of hERG mutations to nonsense-mediated mRNA decay in long QT syndrome. Gene 539: 190-197, 2014.

28. Brown CM, Dalphin ME, Stockwell PA and Tate WP: The translational termination signal database. Nucleic Acids Res 21: 3119-3123, 1993.

29. Brown CM, Stockwell PA, Trotman CN and Tate WP: Sequence analysis suggests that tetra-nucleotides signal the termination of protein synthesis in eukaryotes. Nucleic Acids Res 18: 6339-6345, 1990

30. Bonetti B, Fu L, Moon J and Bedwell DM: The efficiency of translation termination is determined by a synergistic interplay between upstream and downstream sequences in Saccharomyces cerevisiae. J Mol Biol 251: 334-345, 1995.

31. McCaughan KK, Brown CM, Dalphin ME, Berry MJ and Tate WP: Translational termination efficiency in mammals is influenced by the base following the stop codon. Proc Natl Acad Sci USA 92: 5431-5435, 1995.

32. Tate WP, Poole ES, Horsfield JA, Mannering SA, Brown CM, Moffat JG, Dalphin ME, McCaughan KK, Major LL and Wilson DN: Translational termination efficiency in both bacteria and mammals is regulated by the base following the stop codon. Biochem Cell Biol 73: 1095-1103, 1995.

33. Manuvakhova M, Keeling K and Bedwell DM: Aminoglycoside antibiotics mediate context-dependent suppression of termination codons in a mammalian translation system. RNA 6: 1044-1055, 2000.

34. Howard MT, Shirts BH, Petros LM, Flanigan KM, Gesteland RF and Atkins JF: Sequence specificity of aminoglycoside-induced stop codon read-through: Potential implications for treatment of Duchenne muscular dystrophy. Ann Neurol 48: 164-169, 2000.

35. Keeling KM and Bedwell DM: Clinically relevant aminoglycosides can suppress disease-associated premature stop mutations in the IDUA and P53 cDNAs in a mammalian translation system. J Mol Med (Berl) 80: 367-376, 2002.

This work is licensed under a Creative Commons

Attribution-NonCommercial-NoDerivatives 4.0 International (CC BY-NC-ND 4.0) License. 